\title{
Successful treatment of lipofibromatosis-like neural tumor of the lumbar spine with an NTRK-fusion inhibitor
}

\author{
Megan Dupuis ${ }^{1}$ (1), Yulei Shen ${ }^{2}$, Christian Curcio ${ }^{3}$, Jeanne M. Meis ${ }^{3}$, Wei-Lien Wang ${ }^{3}$, Behrang Amini ${ }^{4}$, \\ Laurence Rhines ${ }^{5}$, Jacquelyn Reuther ${ }^{2}$, Angshumoy Roy ${ }^{2}$, Kevin E. Fisher ${ }^{2}$, Anthony P. Conley ${ }^{6}$ \\ and J. Andrew Livingston ${ }^{6,7^{*}}$
}

\begin{abstract}
Background: Lipofibromatosis-like neural tumors (LPF-NT) are a newly identified class of rare mesenchymal neoplasms. Current standard of care therapy is surgical resection alone; there are no chemotherapies or molecular targeted therapies that have been shown to be effective in patients who are not surgical candidates due to either tumor bulk or location. Most LPF-NT harbor NTRK fusions, although the therapeutic significance of these fusions has not been previously demonstrated in this malignancy. Here, we present the first case of a patient with surgically-unresectable LPF-NT successfully treated with medical therapy, specifically the TRK fusion-protein inhibitor entrectinib.

Case presentation: The patient is a 21 year old man with no co-morbidities who presented for evaluation due to intermittent abdominal pain and was found to have a mass spanning from T12-L2. Biopsy revealed a mesenchymal spindle cell neoplasm and S100 positivity pointed to possible nerve sheath origin. The sample was ultimately found to have an LMNA-NTRK1 fusion, confirming the diagnosis of LP-NFT. Unfortunately, due to the bulk and location of the tumor, surgery was felt to be exceptionally morbid and the patient was treated in a clinical trial with the NTRK inhibitor entrectinib. Surprisingly, he had such a robust clinical response that he was ultimately deemed a surgical candidate and he was successfully taken to surgery. Post-operative pathology revealed $>95 \%$ necrosis, demonstrating exceptional sensitivity to the targeted therapy. The patient remains NED and on entrectinib 12 months post-operatively.

Conclusions: The exceptional treatment response of this patient suggests that NTRK fusions are true drivers of the disease. Thus, all patients should be evaluated for NTRK fusions using sensitive methodologies and treatment with TRK fusion-protein inhibitors should be considered in patients who are not candidates for oncologic resection.
\end{abstract}

Keywords: Lipofibromatosis-like neural tumors, LPF-NT, NTRK, Entrectinib

*Correspondence: Jalivingston@mdanderson.org

${ }^{6}$ Department of Sarcoma Medical Oncology, University of Texas MD

Anderson Cancer Center, 1515 Holcombe Blvd. Unit 0450, Houston, TX 77030, USA

Full list of author information is available at the end of the article

\section{Background}

Lipofibromatosis-like neural tumors (LPF-NT) are a rare subset of typically superficial mesenchymal neoplasms initially described in 2016 [1]. While morphologically similar to lipofibromatosis in that they contain spindle cells involving fibroadipose tissue and are positive for CD34 and SMA, they are also positive for S-100 protein which suggests neural differentiation. Importantly, LPFNT typically contain a driver fusion protein involving

c) The Author(s) 2020. This article is licensed under a Creative Commons Attribution 4.0 International License, which permits use, sharing, adaptation, distribution and reproduction in any medium or format, as long as you give appropriate credit to the original author(s) and the source, provide a link to the Creative Commons licence, and indicate if changes were made. The images or other third party material in this article are included in the article's Creative Commons licence, unless indicated otherwise in a credit line to the material. If material is not included in the article's Creative Commons licence and your intended use is not permitted by statutory regulation or exceeds the permitted use, you will need to obtain permission directly from the copyright holder. To view a copy of this licence, visit http://creativeco mmons.org/licenses/by/4.0/. The Creative Commons Public Domain Dedication waiver (http://creativecommons.org/publicdomain/ zero/1.0/) applies to the data made available in this article, unless otherwise stated in a credit line to the data. 
TRK, a family of neurotrophic receptor tyrosine kinases [1]. This molecular alteration distinguishes this neoplasm from lipofibromatosis, which does not harbor a TRK fusion protein [1]. These tumors are locally invasive and can carry significant morbidity [1]. Currently, front-line therapy for LPF-NT is surgery alone; if resection is not an option due to tumor bulk or location, there are no validated standardized treatments. Thus, a lack of effective pre-surgical therapies represents a significant gap in the field for this newly-described tumor.

In recent years, cancer treatments have dramatically changed due to identification of new, druggable, oncogenic molecular drivers. One such target is the fusion product of the neurotrophic receptor tyrosine kinase genes NTRK1, NTRK2, and NTRK3 (encoding proteins TRK1, TRK2, and TRK3, respectively) with a variety of genetic fusion partners [2]. These upstream fusion partners contain oligomerization domains (such as coiledcoil, zinc finger, or WD domains) [3, 4] or have alternate mechanisms of dimerization which activate TRK downstream signaling; more than 50 upstream partners have been identified thus far [5].

$N T R K$ fusions have been identified in tumors of more than 20 histologies [5]. Certain NTRK fusions, like ETV6NTRK3, are represented in $>90 \%$ of secretory breast carcinomas [6], mammary analog of secretory carcinomas (MASC) [7], congenital mesoblastic nephroma [8, 9], and infantile fibrosarcomas [10, 11]. These fusions are also found at lower incidences (5-25\%) in tumors such as breast, lung, colon, and melanoma [2], leading to the 2018 basket trial in which 55 NTRK-fusion-positive patients with 17 different cancer types were treated with the first-generation TRK inhibitor larotrectinib [12]. This study ultimately resulted in the first tissue-agnostic US FDA approval for a molecular targeted therapy. Entrectinib, another first generation small-molecule inhibitor against TRKA/B/C, ROS1, and ALK, was recently developed. It was designed to cross the blood-brain barrier to target brain metastases [13], and demonstrated efficacy in multiple histologies including non-small cell lung cancer $[14,15]$ as well as activity in adults and children with solid tumors harboring NTRK fusions [16]. These studies have led to its accelerated approval by the US FDA for adults with ROS-1 positive metastatic NSCLC and for adult and pediatric patients $\geq 12$ years old with NTRK fusion-positive solid tumors.

Thus far, two papers have studied the incidence of NTRK fusion proteins in LPF-NT. In the 2016 paper which first classified this tumor [1], they describe that $10 / 14$ patients $(71 \%)$ had NTRK 1 fusions. In the second study [17], molecular studies showed $4 / 5$ patients with LPF-NF were positive for NTRK1 rearrangement by FISH. Given the natural history and therapeutic implications of NTRK fusions in a subset of soft tissue sarcomas, the WHO has recently reclassified all NTRKrearranged soft tissue sarcoma into a new provisional entity labeled NTRK-rearranged spindle cell neoplasm [18]. This new classification encompasses LPF-NT as well as other sarcomas harboring NTRK fusions. This move towards molecularly defined subtypes of soft tissue sarcoma is reflective of the field and the evolving role of subtype specific diagnoses and treatments. However, the significance and therapeutic implications of NTRKfusions across various sarcoma subtypes may not be uniform and thus warrants specific evaluation as in our case of LPF-NT.

Taken together, the above studies suggest that most LPF-NT harbor an NTRK fusion protein, a viable therapeutic target. Here, we report the first case of an LPF-NT successfully treated with a TRK inhibitor prior to surgical resection, and discuss the implications for management of this rare entity.

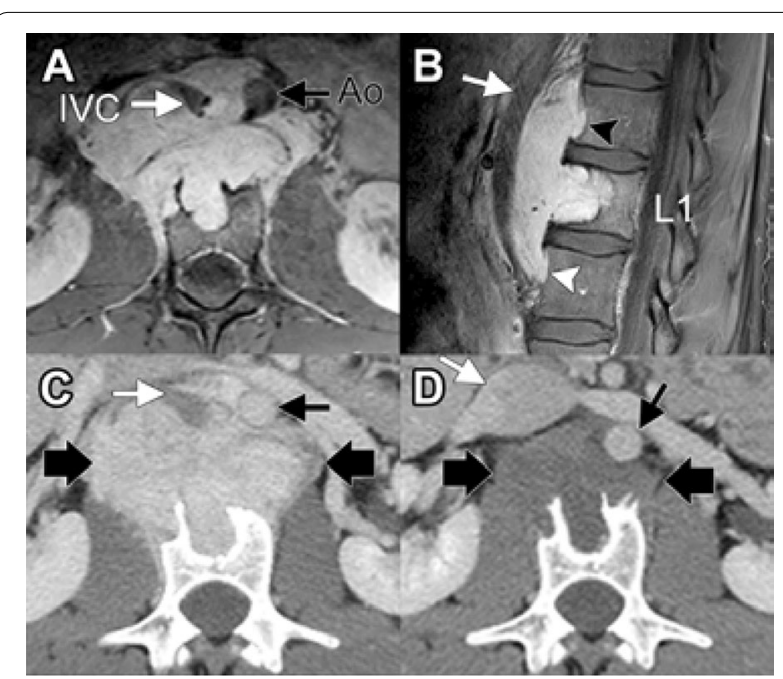

Fig. 1 Imaging features of the lesion. $\mathbf{a}, \mathbf{b}$ Axial and sagittal post-contrast MRI demonstrates an avidly enhancing lesion in the $\mathrm{L} 1$ pre-vertebral soft tissues. There is anterior displacement of the aorta (Ao, black arrow) and inferior vena cava (IVC, white arrows). There is invasion of the L1 vertebral body, with cephalad and caudad extension of disease and secondary pressure erosion of the ventral cortices of T12 (black arrowhead) and L2 (white arrowhead) vertebral bodies. c Concurrent contrast-enhanced CT image at the level of $\mathrm{L} 1$ shows an avidly enhancing mass (between large black arrows) with invasion of the $L 1$ vertebral body. The CT shows rim of sclerosis in the vertebral body indicative of secondary invasion from a soft tissue mass. The aorta and inferior vena cava (black and white arrows, respectively) are anteriorly displaced. $\mathbf{d}$ Contrast-enhanced $\mathrm{CT}$ at end of therapy shows significant decrease in size and degree of enhancement of the mass (between large black arrows). Mass effect on the aorta and inferior vena cava (black and white arrows, respectively) has also decreased 


\section{Case presentation}

The patient is a 21-year-old young man without significant past medical history. He initially presented with a complaint of intermittent abdominal pain, and a CT scan revealed an abnormal paraspinal lesion. He underwent MRI which demonstrated the lesion extending from T12L2 and measured $8.7 \mathrm{~cm} \times 3.9 \mathrm{~cm} \times 6.9 \mathrm{~cm}$ (Fig. 1a, b).

Subsequent biopsy revealed a spindle cell mesenchymal tumor with monotonous, bland spindle cells admixed with mature fat and occasional ectatic blood vessels (Fig. 2). Neither necrosis nor brisk mitotic activity were noted. IHC studies demonstrated positivity for CD34, pan-TRK, S-100 and SMA (not shown); the tumor was negative for Pankeratin cocktail, STAT6, DOG1, desmin, TLE1, panmelanocytic cocktail, and Sox10. Scattered cells labeled for SATB2 and H3K27me3. The tumor was negative for $M D M 2$ amplification or FUS gene rearrangement using fluorescent in situ hybridization (FISH).

The S-100 staining raised the possibility of a peripheral nerve sheath origin. Given the pan-TRK immunoreactivity, the diagnosis of LPF-NT was considered; however, the site was unusual as it was not superficial. Thus, further molecular testing was performed. A custom-designed, clinically-validated anchored multiplex
PCR-based targeted next-generation sequencing (NGS) RNA fusion panel that covers 485 exons from 81 genes and is optimized for formalin-fixed, paraffin-embedded (FFPE) samples, revealed a fusion between exon 2 of LMNA (NM_005572.3; chr1:156100564) and exon 11 of NTRK1 (NM_002529.3; chr1:156844698), predicted to encode an in-frame LMNA-NTRK1 fusion protein retaining the C-terminal kinase domain of NTRK1 (Fig. 3a). The fusion transcripts were confirmed with orthogonal RT-PCR and Sanger sequencing (Fig. 3b). Identification of this fusion reclassified the putative diagnosis to an S-100 positive LPF-NT of the spine.

Due to size and location, primary surgical resection would have been exceedingly morbid. Thus, the patient was entered into a phase II basket trial enrolling patients with $N T R K 1 / 2 / 3$, ROS1, or ALK gene rearrangements to be treated with entrectinib. He received a dose of $600 \mathrm{mg}$ daily, and the only adverse effects were grade 1 diarrhea and taste alterations. Early response assessment after 1 month demonstrated a near-complete loss of enhancement and density of the mass, with the hounsfield units dropping from 200 on the initial scan to 55-60 at follow up (Figs. 1a, b, and 4). $\mathrm{He}$ had an excellent therapeutic response, with a $45 \%$

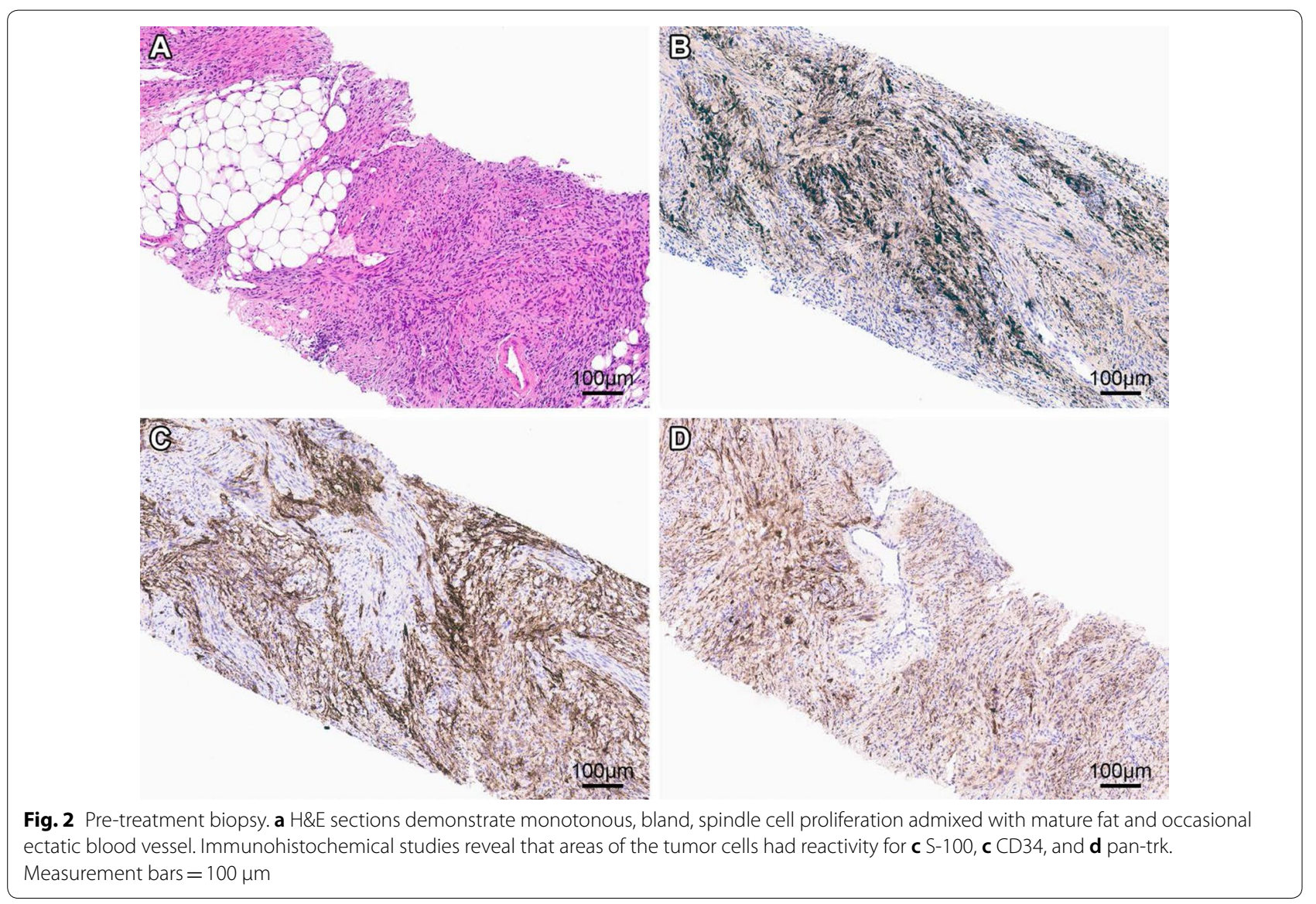




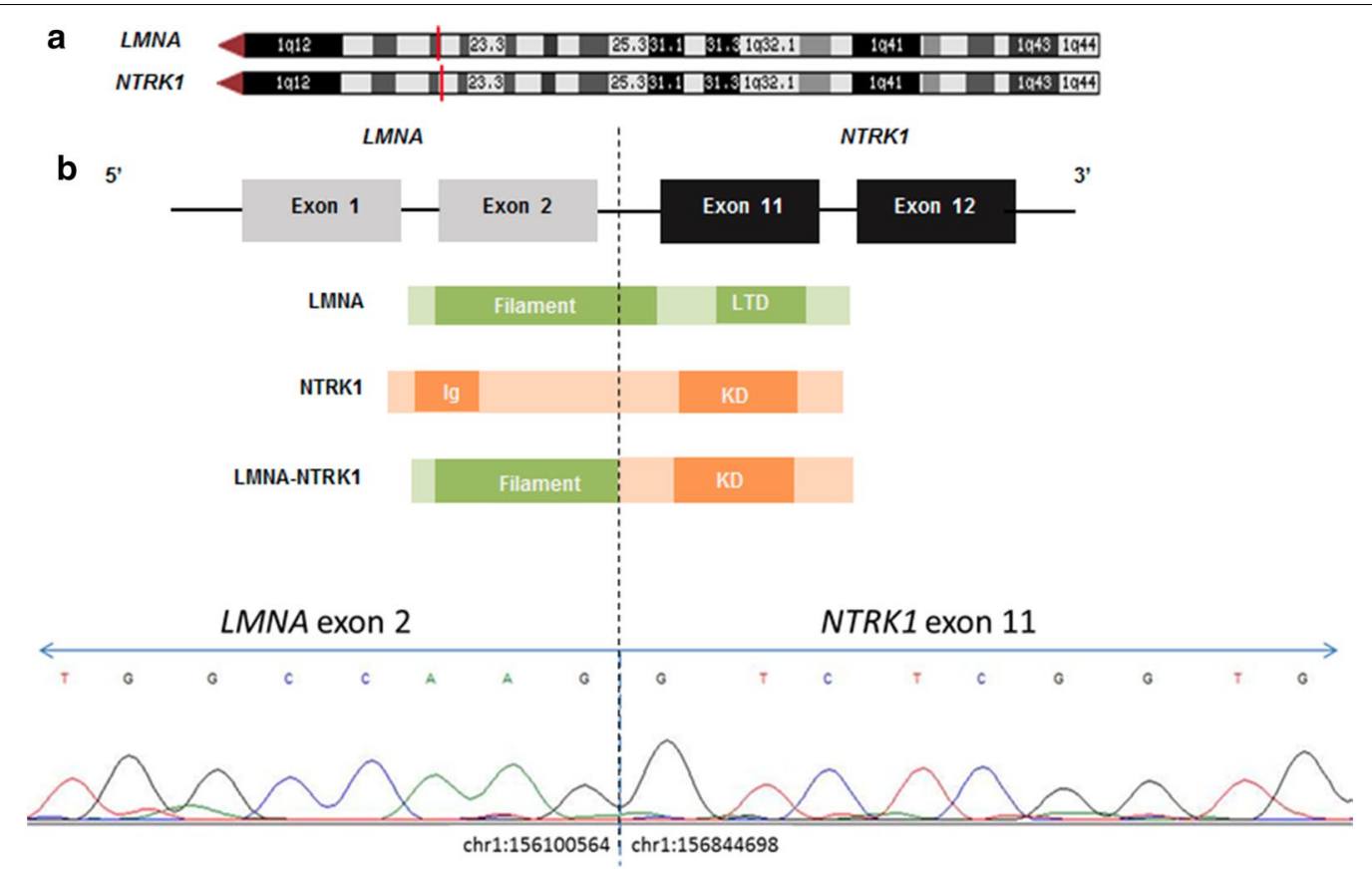

Fig. 3 LMNA-NTRK1 fusion confirmed by RT-PCR. a Schematic diagram showing the predicted in-frame LMNA-NTRK1 fusion protein joining the $5^{\prime}$ LMNA filament domain to an intact 3' NTRK1 tyrosine kinase domain (KD). The red dashed lines denote the 1q chromosomal positions of LMNA and NTRK1, respectively. b RT-PCR Sanger sequencing trace confirming the fusion breakpoint at chr1:156100564 (LMNA, NM_005572.3, exon 2) and chr1:156844698 (NTRK1, NM_002529.3, exon 11). LTD, lamin tail domain; Ig, Tyrosine-protein kinase receptor C2 lg-like domain
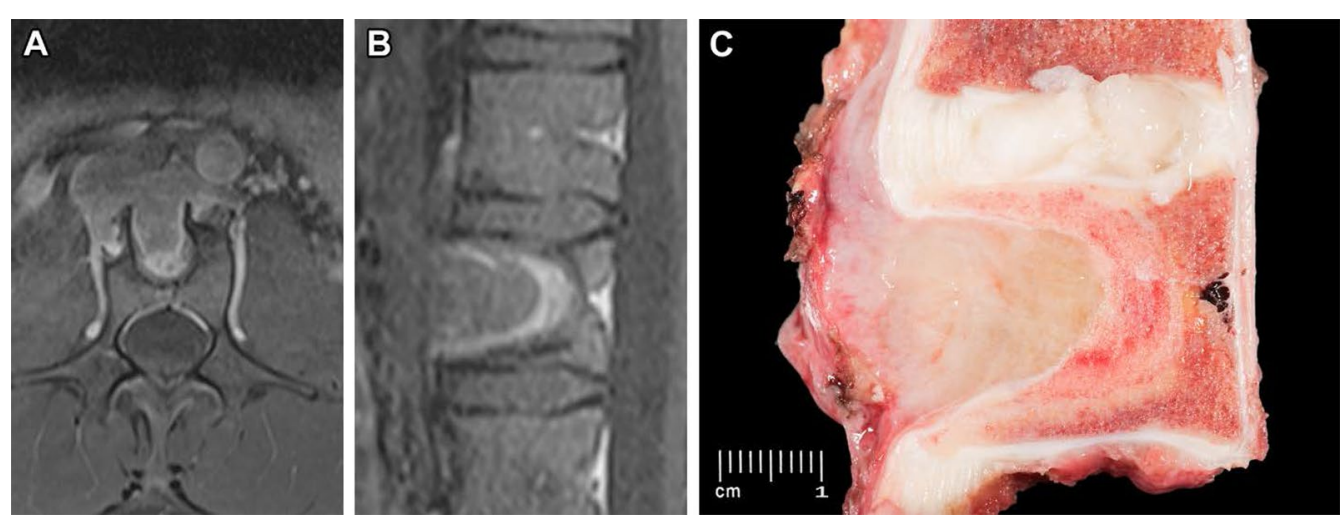

Fig. 4 Post treatment. Contrast-enhanced MR images demonstrating mass involving vertebrae (a) axial and (b) sagittal showing decrease in the size of the lesion compared to baseline (Fig. 1). c Gross specimen (sagittal) demonstrating a tan-white pink mass involving the paraspinal soft tissue and protruding into the underlying vertebrae body associated with sclerosis. Measurement bar $=1 \mathrm{~cm}$

reduction in tumor size by RECIST criteria, measuring $4.0 \times 2.8 \mathrm{~cm}$ (Fig. 5). Although the patient was not initially a surgical candidate due to unacceptable morbidity, his response to therapy was so exceptional that he ultimately qualified for surgical resection.

Therefore, he underwent vertebrectomy, which revealed a $7.0 \times 5.2 \times 3 \mathrm{~cm}$ mass with tan-white pink cut surface involving paraspinal soft tissue and protruding into the vertebral body, with underlying bony sclerosis. Histologically, there was extensive treatment response $(>95 \%)$ with decreased cellularity, marked hyalinization and focal areas of viable tumor cells (Fig. 6). The tumor focally involved soft tissue margins however bone margins were tumor-free. The patient has continued on entrectinib following surgery and remains without evidence of recurrence at 7 months. 


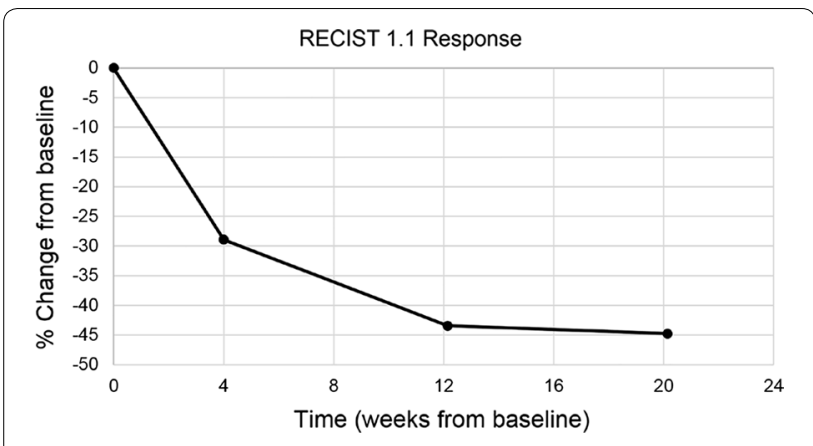

Fig. 5 Response to therapy as assessed by RECIST 1.1. Response plateau was achieved at week 12 , with $45 \%$ decrease in size of the lesion

\section{Discussion and conclusions}

LPF-NT is a rare tumor of mesenchymal origin first described in 2016. Prior to then, LPF-NT was likely characterized as atypical lipofibromatosis, malignant peripheral nerve sheath tumor (MPNST), or spindle cell tumors. Given its recent classification, there is a paucity of data regarding management and outcomes. Currently, only 26 patients with LPF-NT are described in the literature $[1,17,19]$; our case, which met classification criteria for LPF-NT given positive S100 and CD34 staining, represents the 27th patient.

Importantly, his tumor was positive for a LMNANTRK1 fusion, which was the most common type of NTRK fusion seen in the initial case series [1]. The authors also separately report a single case of a patient with LPF-NT and LMNA-NTRK1 fusion with lung metastasis, potentially secondary to a delay in surgical resection rather than the specific NTRK fusion partner. Another case report has also implicated the $L M N A$ NTRK1 fusion in a patient with metastatic sarcoma [20]. However, the significance of specific NTRK fusion partners remains incompletely understood $[2,5]$, and their systematic classification may yield information about their biologic behavior.

In the broader sarcoma field, the significance of NTRK fusions has emerged. As previously mentioned, ETV6NTRK3 fusions are practically pathognomonic for the diagnosis of infantile fibrosarcoma [11] and NTRK fusions have also been implicated as the defining feature of a subset of unclassified uterine sarcoma with spindle cell morphology [21]. However, incidence of NTRK fusions is variable between different sarcoma subtypes. Recently, position papers released from the Journal of Clinical Pathology [22] and ESMO [23] propose standardized algorithms for testing for NTRK fusions. Both propose essentially the same method: for tumors with a high incidence of NTRK fusions (such as MASC), any detection method is sufficient; however, in tumors with low incidence, an NGS panel should be used upfront with positivity confirmed via IHC. If, however, no standard NGS panel is available, then IHC screening may be used upfront, with NGS confirmation.

For poor quality specimens, a highly sensitive and versatile assay able to test for NTRK and other fusions is needed to confirm the diagnosis. Anchored multiplex PCR NGS allows for detection of multiple gene fusions in a single assay with minimal RNA input, which has high diagnostic yield in sarcomas and other spindle cell lesions [24].

As these fusions have been identified, the opportunity for targeted therapy has also become apparent. In 2015, a patient with metastatic soft-tissue sarcoma harboring an $L M N A-N T R K 1$ fusion protein was enrolled phase I clinical trial with larotrectinib and had nearly complete regression of the lung tumors. In the 2018 basket trial evaluating larotrectinib, 21/55 patients had sarcomas and all but 2 experienced at least a partial response [12]. In 2018, an integrated analysis of two phase I clinical trials
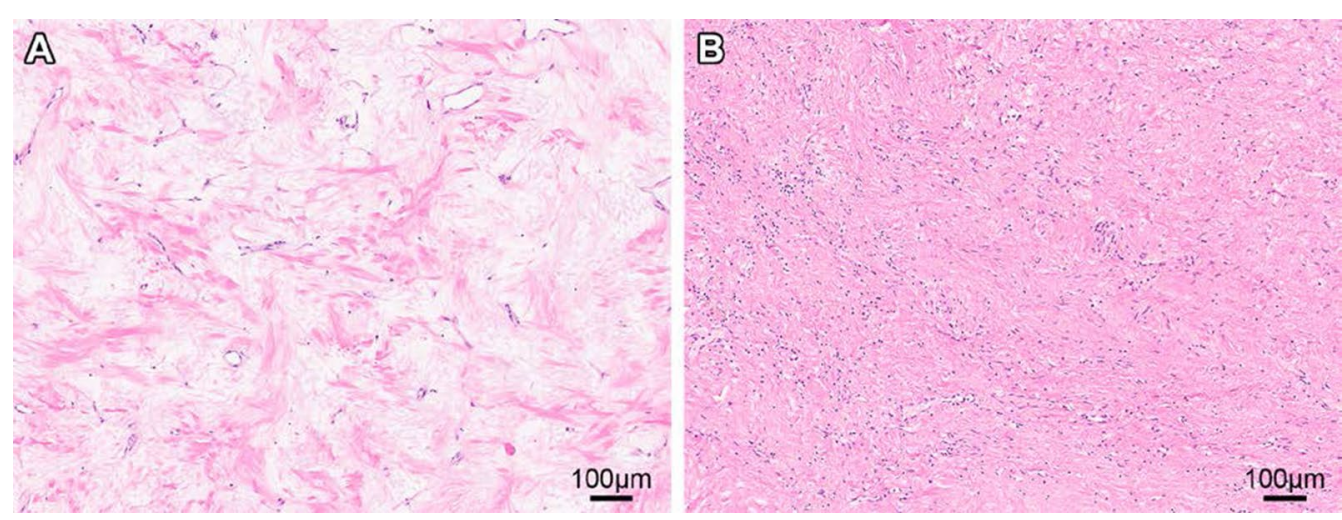

Fig. 6 Post-treatment histological changes. a H\&E sections demonstrate marked hyalinization and decreased cellularity with scattered foci of $\mathbf{b}$ residual tumor cells admixed with lymphocytes. Measurement bars $=100 \mu \mathrm{m}$ 
and one phase II clinical trial revealed that entrectinib had a 57\% ORR among 54 patients with NTRK fusions [15]. Entrectinib was well tolerated, produced durable systemic responses, and was FDA-approved based on these data.

In LPF-NT, the frontline therapy is surgical resection. However, in patients for whom surgery is not an option due to location or bulk of tumor, no chemotherapies or molecular therapies have successfully reduced tumor burden. To our knowledge, this case represents the first successful treatment of LPF-NT with an NTRK-fusion inhibitor prior to surgery. Our patient had an excellent response to entrectinib, allowing him to proceed to surgical resection. Importantly, post-operative pathology revealed $>95 \%$ necrosis, consistent with exquisite inhibitor sensitivity. These results suggest that all patients with a presumed diagnosis of LPF-NT should be screened for NTRK fusions using sensitive methodologies, and that treatment with a TRK fusion-protein inhibitor is a rational therapeutic option for patients who are not up-front surgical candidates.

\section{Abbreviations}

LPF-NT: Lipofibromatosis-like neural tumor; NTRK: Neurotrophic receptor tyrosine kinase; MASC: Mammary analog of secretory carcinoma; NSCLC: Nonsmall cell lung cancer; NGS: Next generation sequencing; MPNST: Malignant peripheral nerve sheath tumor.

\section{Acknowledgements}

Not applicable.

\section{Authors' contributions}

MD participated in conception of the report and the major drafting of the manuscript as well as all revisions; YS, CC, JM, W-LW, JR, AR, KF, acquired and interpreted data in regards to the immunohistochemistry and molecular testing of the sample leading to the accurate diagnosis. BA acquired and interpreted imaging data including RECIST criteria; LR performed the surgery and participated in the conception of the manuscript; AC participated in conception of the manuscript, data analysis, and was a major contributor to the manuscript; JAL conceived of the manuscript, data analysis, and was a major contributor to the manuscript and subsequent revisions of the manuscript. All authors read and approved the final manuscript.

\section{Funding}

No funding was directly used to write or prepare this case report.

\section{Availability of data}

Not applicable.

\section{Ethics approval and consent to participate}

Enrollment of the patient in a clinical trial was completed in compliance with our institution's ethics committee and institutional review board.

\section{Consent for publication}

Consent for publication was obtained from the patient using our institution's consent policy and will be provided upon request.

\section{Competing interests}

AC receives research funding from Ignyta and Genentech for the conduct of the entrectinib study, and provides consulting for Genentech and scientific advisement for Deciphera; no other authors have competing interests to declare.

\section{Author details}

${ }^{1}$ Division of Cancer Medicine, University of Texas MD Anderson Cancer Center, Houston, TX, USA. ${ }^{2}$ Department of Pathology \& Immunology, Baylor College of Medicine and Texas Children's Hospital, Houston, TX, USA. ${ }^{3}$ Department of Pathology, University of Texas MD Anderson Cancer Center, Houston, TX, USA. ${ }^{4}$ Department of Diagnostic Imaging, University of Texas MD Anderson Cancer Center, Houston, TX, USA. ${ }^{5}$ Department of Neurosurgery, University of Texas MD Anderson Cancer Center, Houston, TX, USA. ${ }^{6}$ Department of Sarcoma Medical Oncology, University of Texas MD Anderson Cancer Center, 1515 Holcombe Blvd. Unit 0450, Houston, TX 77030, USA. ${ }^{7}$ Department of Pediatrics, University of Texas MD Anderson Cancer Center, Houston, TX, USA.

Received: 9 April 2020 Accepted: 30 July 2020

Published online: 06 August 2020

\section{References}

1. Agaram NP, Zhang L, Sung YS, et al. Recurrent NTRK1 gene fusions define a novel subset of locally aggressive lipofibromatosis-like neural tumors. Am J Surg Pathol. 2016:40:1407-16.

2. Amatu A, Sartore-Bianchi A, Siena S. NTRK gene fusions as novel targets of cancer therapy across multiple tumour types. ESMO Open. 2016;1:e000023.

3. Coulier F, Martin-Zanca D, Ernst M, et al. Mechanism of activation of the human trk oncogene. Mol Cell Biol. 1989;9:15-23.

4. Schram AM, Chang MT, Jonsson P, et al. Fusions in solid tumours: diagnostic strategies, targeted therapy, and acquired resistance. Nat Rev Clin Oncol. 2017;14:735-48.

5. Cocco E, Scaltriti M, Drilon A. NTRK fusion-positive cancers and TRK inhibitor therapy. Nat Rev Clin Oncol. 2018;15:731-47.

6. Tognon C, Knezevich SR, Huntsman D, et al. Expression of the ETV6NTRK3 gene fusion as a primary event in human secretory breast carcinoma. Cancer Cell. 2002;2:367-76.

7. Drilon A, Li G, Dogan S, et al. What hides behind the MASC: clinical response and acquired resistance to entrectinib after ETV6-NTRK3 identification in a mammary analogue secretory carcinoma (MASC). Ann Oncol. 2016;27:920-6.

8. Davis JL, Lockwood CM, Albert CM, et al. Infantile NTRK-associated mesenchymal tumors. Pediatr Dev Pathol. 2018;21:68-78.

9. Halalsheh $\mathrm{H}, \mathrm{McC}$ arville $\mathrm{MB}$, Neel M, et al. Dramatic bone remodeling following larotrectinib administration for bone metastasis in a patient with TRK fusion congenital mesoblastic nephroma. Pediatr Blood Cancer. 2018;65:e27271.

10. Pavlick D, Schrock AB, Malicki D, et al. Identification of NTRK fusions in pediatric mesenchymal tumors. Pediatr Blood Cancer. 2017;64:e26433.

11. Laetsch TW, DuBois SG, Mascarenhas L, et al. Larotrectinib for paediatric solid tumours harbouring NTRK gene fusions: phase 1 results from a multicentre, open-label, phase 1/2 study. Lancet Oncol. 2018;19:705-14.

12. Drilon A, Laetsch TW, Kummar S, et al. Efficacy of larotrectinib in TRK fusion-positive cancers in adults and Children. N Engl J Med. 2018;378:731-9.

13. Ardini E, Menichincheri M, Banfi P, et al. Entrectinib, a Pan-TRK, ROS1, and ALK inhibitor with activity in multiple molecularly defined cancer indications. Mol Cancer Ther. 2016;15:628-39.

14. Farago $A F$, Le $L P$, Zheng $Z$, et al. Durable clinical response to entrectinib in NTRK1-rearranged non-small cell lung cancer. J Thorac Oncol. 2015;10:1670-4.

15. Demetri GD, Paz-Ares $L$, Farago AF, et al. Efficacy and safety of entrectinib in patients with NTRK fusion-positive tumors: pooled analysis of STARTRK-2, STARTRK-1 and ALKA-372-001. Munich: ESMO Congress; 2018.

16. Robinson GW, Gajjar AJ, Gauvain KM, et al. Phase 1/1B trial to assess the activity of entrectinib in children and adolescents with recurrent or refractory solid tumors including central nervous system (CNS) tumors. Am Soc Clin Oncol. 2019;33(15):10009.

17. Lao IW, Sun M, Zhao M, et al. Lipofibromatosis-like neural tumour: a clinicopathological study of ten additional cases of an emerging novel entity. Pathology. 2018;50:519-23.

18. WHO. Classification of tumors: soft tissue and bone tumors. 5th ed. Geneva:WHO; 2020. 
19. Bartenstein DW, Coe TM, Gordon SC, et al. Lipofibromatosis-like neural tumor: case report of a unique infantile presentation. JAAD Case Rep. 2018:4:185-8.

20. Wong V, Pavlick D, Brennan T, et al. Evaluation of a congenital infantile fibrosarcoma by comprehensive genomic profiling reveals an LMNA-NTRK1 gene fusion responsive to crizotinib. J Natl Cancer Inst. 2016;108(1):307. https://doi.org/10.1093/jnci/djv307.

21. Chiang S, Cotzia P, Hyman DM, et al. NTRK fusions define a novel uterine sarcoma subtype with features of fibrosarcoma. Am J Surg Pathol. 2018:42:791-8.

22. Penault-Llorca F, Rudzinski ER, Sepulveda AR. Testing algorithm for identification of patients with TRK fusion cancer. J Clin Pathol. 2019;72:460-7.
23. Marchio C, Scaltriti M, Ladanyi M, et al. ESMO recommendations on the standard methods to detect NTRK fusions in daily practice and clinical research. Ann Oncol. 2019;30:1417-27.

24. Lam SW, Cleton-Jansen AM, Cleven AHG, et al. Molecular analysis of gene fusions in bone and soft tissue tumors by anchored multiplex PCR-based targeted next-generation sequencing. J Mol Diagn. 2018;20:653-63.

\section{Publisher's Note}

Springer Nature remains neutral with regard to jurisdictional claims in published maps and institutional affiliations.
Ready to submit your research? Choose BMC and benefit from:

- fast, convenient online submission

- thorough peer review by experienced researchers in your field

- rapid publication on acceptance

- support for research data, including large and complex data types

- gold Open Access which fosters wider collaboration and increased citations

- maximum visibility for your research: over $100 \mathrm{M}$ website views per year

At BMC, research is always in progress.

Learn more biomedcentral.com/submissions 